\title{
Melatonin administration can entrain the free-running circadian system of blind subjects
}

\author{
S W Lockley, D J Skene, K James, K Thapan, J Wright and J Arendt \\ School of Biological Sciences, University of Surrey, Guildford, Surrey GU2 5XH, UK \\ (Requests for offprints should be addressed to S W Lockley)
}

\begin{abstract}
Although melatonin treatment has been shown to phase shift human circadian rhythms, it still remains ambiguous as to whether exogenous melatonin can entrain a free-running circadian system. We have studied seven blind male subjects with no light perception who exhibited free-running urinary 6sulphatoxymelatonin (aMT6s) and cortisol rhythms.

In a single-blind design, five subjects received placebo or $5 \mathrm{mg}$ melatonin p.o. daily at $2100 \mathrm{~h}$ for a full circadian cycle (35-71 days). The remaining two subjects also received melatonin (35-62 days) but not placebo. Urinary aMT6s and cortisol $(n=7)$ and core body temperature $(n=1)$ were used as phase markers to assess the effects of melatonin on the circadian system.
\end{abstract}

During melatonin treatment, four of the seven free-running subjects exhibited a shortening of their cortisol circadian period (tau). Three of these had taus which were statistically indistinguishable from entrainment. In contrast, the remaining three subjects continued to free-run during the melatonin treatment at a similar tau as prior to and following treatment. The efficacy of melatonin to entrain the free-running cortisol rhythms appeared to be dependent on the circadian phase at which the melatonin treatment commenced.

These results show for the first time that daily melatonin administration can entrain free-running circadian rhythms in some blind subjects assessed using reliable physiological markers of the circadian system.

Journal of Endocrinology (2000) 164, R1-R6

\section{Introduction}

The ability of the pineal hormone melatonin (N-acetyl 5methoxytryptamine) to phase shift the circadian system has been extensively investigated in humans. The first demonstration of such an effect was by Arendt and co-workers (1985a) who showed a phase advance (1-3 h) of the endogenous plasma melatonin rhythm in 5 out of 11 subjects following $2 \mathrm{mg}$ melatonin administered daily at $1700 \mathrm{~h}$ for 3 weeks. Subsequently, human phase response curves (PRC) to melatonin have been constructed using various melatonin doses and regimes (Lewy et al. 1992, Zaidan et al. 1994, Lewy \& Sack 1997, Middleton et al. 1997, Lewy et al. 1998). Generally, the studies concur, that when melatonin is administered in the late subjective day/early subjective night, phase advances are induced and when given in the late subjective night/early subjective day, phase delays occur. However, in humans, it is difficult to achieve the steady-state free-running circadian rhythms which are a prerequisite to assess phase shifts and to construct a PRC. Unfortunately, the variety of approaches, the different physiological markers and definitions of phase used to construct PRCs have led to some ambiguity as to its exact shape.

Although melatonin has been shown to phase shift the human circadian system, there have been few reports of entrainment by melatonin in humans. Evidence of entrainment of core body temperature (CBT), hormonal and sleep-wake rhythms by melatonin treatment has been shown in some sighted (Middleton et al. 1997, Hashimoto et al. 1998, Hayakawa et al. 1998) and blind adults (Arendt et al. 1988) with free-running rhythms. Sack and colleagues (Sack et al. 1987, 1990, 1991) have shown cumulative phase advances of the endogenous melatonin and cortisol rhythms of up to $8.4 \mathrm{~h}$ in free-running blind males following daily melatonin administration $(0.5 \mathrm{mg}$ and $5 \mathrm{mg})$ at $2200 \mathrm{~h}$ or bedtime for up to 21 days. The claim that a free-running blind man exhibited entrainment following several years of self-medication of melatonin (Sack et al. 1990, 1991) has recently been questioned (Sack \& Lewy 1997).

Despite the lack of evidence for consistent entrainment of physiological rhythms in humans, several studies have shown stabilisation of the sleep-wake rhythm and improved sleep in blind adults during melatonin treatment (Arendt et al. 1988, Aldhous \& Arendt 1991, Tzischinsky et al. 1992, Arendt et al. 1997). Similar improvements have been shown in sighted free-running subjects (Kato et al. 1998, Siebler et al. 1998) but not in all cases (Okawa et al. 1998). However, in the blind only one of these studies assessed the effect of melatonin administration on a circadian phase marker (Folkard et al. 1990). In addition, the strong homeostatic influence on sleep (Borbély 1982) prevents the assumption that other circadian rhythms were similarly stabilised. 
Table 1 Subject details

\begin{tabular}{|c|c|c|c|c|c|c|c|}
\hline $\begin{array}{l}\text { Subject } \\
\text { no. }\end{array}$ & Age & Diagnosis & $\begin{array}{l}\text { No. of intact } \\
\text { eyes }\end{array}$ & $\begin{array}{l}\text { Onset of visual } \\
\text { loss }\end{array}$ & $\begin{array}{l}\text { Rapidity of } \\
\text { visual loss }\end{array}$ & $\begin{array}{l}\text { Melatonin start } \\
\text { date }\end{array}$ & $\begin{array}{l}\text { No. of days } \\
\text { melatonin }\end{array}$ \\
\hline $\mathrm{S} 18$ & 45 & $\begin{array}{l}\text { Retinopathy of } \\
\text { prematurity }\end{array}$ & 0 & Birth & - & $25 \mathrm{Mar}$ & 35 \\
\hline S45 & 43 & Ocular trauma & 0 & 1986 & Days & $2 \mathrm{Nov}$ & 48 \\
\hline S51 & 60 & Retinoblastoma & 1 & Birth & Years & $21 \mathrm{Nov}$ & 60 \\
\hline $\mathrm{S} 17$ & 47 & Microphthalmia & 2 & 1965 & Years & 5 Feb & 71 \\
\hline S31 & 33 & Retinoblastoma & 0 & 1965 & Years & 3 Jun & 35 \\
\hline S26 & 46 & Buphthalmos & 1 & Birth & - & $28 \mathrm{Jul}$ & 62 \\
\hline S62 & 38 & Retinoblastoma & 0 & 1961 & Years & 13 Oct & 35 \\
\hline
\end{tabular}

-, not applicable.

The aims of our study were firstly to evaluate the repeatability of tau measurements in field studies of freerunning blind subjects. Secondly, to assess the ability of exogenous melatonin to entrain the circadian system of freerunning blind subjects. The protocol enables the effect of melatonin to be assessed during a stable, long-term free-run without any influence of the light-dark cycle, the strongest entrainment signal in humans.

\section{Materials and Methods}

\section{Subjects}

Seven male subjects were studied (Table 1). The study was approved by the University of Surrey Advisory Committee on Ethics and the subjects gave informed consent. Subjects were instructed not to take any medications that could affect melatonin and/or the sleep-wake cycle and urine tests for drugs of abuse prior to, during and post-melatonin treatment were all negative.

Five of the subjects had been previously shown to have free-running 6-sulphatoxymelatonin (aMT6s) rhythms (Lockley et al. 1997). The two remaining subjects (S51, S62) were confirmed as having a free-running aMT6s rhythm prior to commencing the melatonin treatment

\section{Parameters measured}

Subjects were assessed for 12-27 weeks. At 1-2 weekly intervals, subjects collected sequential 4-hourly (8-hourly overnight) urine samples for $24(n=1)$ or $48 \mathrm{~h}(n=6)$ for the assessment of aMT6s, the major urinary metabolite of melatonin, and urinary cortisol. Both rhythms are considered reliable markers of circadian phase and have been shown to run in parallel in free-running blind subjects in both laboratory (Sack et al. 1992) and field (Skene et al. 1999) conditions. During melatonin treatment periods, cortisol was used as the marker of circadian phase. During the urine sampling, one subject (S45) also measured CBT continuously via a rectal temperature probe.

\section{Treatment}

In a single blind design, five of the seven subjects were treated daily for one full circadian cycle with $5 \mathrm{mg}$ melatonin p.o. (Penn Pharmaceuticals, Tredegar, UK) or placebo (identical lactose-filled gelatin capsule) at $2100 \mathrm{~h}$. The remaining two subjects (S26, S62) also received melatonin for a full circadian cycle but these data were compared with a no-treatment baseline. Table 1 shows the duration and time of year of the melatonin treatment.

\section{Rhythm analysis}

The aMT6s (ng/h), cortisol (nmol/h) and CBT rhythms were subjected to cosinor analysis (Nelson et al. 1979) to determine the acrophase times of the rhythms. Acrophase times (phi $(\varphi)$ ) were accepted if the probability of fit (p) was $<0.05$ for aMT6s and CBT and $<0.20$ for cortisol. To assess the period of the aMT6s, cortisol and CBT rhythm, acrophases were fitted with best-fit regression lines (tau $(\tau)=24+$ slope) (Lockley et al. 1997, Skene et al. 1999) during the pre-melatonin treatment period, the melatonin treatment and the post-melatonin period. The mid-point of the upward and downward mean crossing times (Shanahan et al. 1999) were also subjected to regression analysis. The parameters were considered free-running if the period of the rhythm was different to $2400 \mathrm{~h}$ and the $95 \%$ confidence limits of the regression line did not encompass 0 (i.e. $2400 \mathrm{~h}$ ) and entrained if the period of the rhythm was close to $2400 \mathrm{~h}$ and the $95 \%$ limits did cross 0 . Placebo and notreatment data were analysed together. The overall tau was taken from the longest sequence of urine data without melatonin treatment.

\section{Assays}

The urinary aMT6s rhythm was measured by radioimmunoassay (RIA) (Arendt et al. 1985b, Aldhous \& Arendt 
Table 2 Tau analysis for urinary aMT6s and cortisol rhythms before, during and after treatment with melatonin in subjects who were a) not entrained by melatonin and b) entrained by melatonin

\begin{tabular}{lllll} 
Pre-melatonin & \multicolumn{5}{c}{ During melatonin } & Post-melatonin \\
\hline $\begin{array}{l}\text { aMT6s } \\
\text { tau (h) } \\
( \pm 95 \% \text { limits })\end{array}$ & $\begin{array}{l}\text { Cortisol } \\
\text { tau }(\mathrm{h}) \\
( \pm 95 \% \text { limits })\end{array}$ & $\begin{array}{l}\text { Cortisol } \\
\text { tau }(\mathrm{h}) \\
( \pm 95 \% \text { limits })\end{array}$ & $\begin{array}{l}\text { aMT6s } \\
\text { tau }(\mathrm{h}) \\
( \pm 95 \% \text { limits })\end{array}$ & $\begin{array}{l}\text { Cortisol } \\
\text { tau (h) } \\
( \pm 95 \% \text { limits })\end{array}$ \\
\hline & & & & \\
- & - & $24.70 \pm 0.56$ & $24.72 \pm 0.84$ & $24.96 \pm 0.61$ \\
$24.52 \pm 0.34$ & $24.87 \pm 0.21$ & $25.22 \pm 2.59$ & $24.91 \pm 0.20$ & $24.97 \pm 0.11$ \\
$24.49 \pm 0.12$ & $24.53 \pm 0.09$ & $24.52 \pm 2.24$ & - & - \\
& & & & \\
- & & & $24.27 \pm 0.08$ & $24.30 \pm 0.06$ \\
$24.57 \pm 0.24$ & $24.57 \pm 0.23$ & $24.03 \pm 0.20$ & - & - \\
24.43 & 24.45 & $24.05 \pm 0.80$ & 24.28 & - \\
$24.81 \pm 0.13$ & $24.80 \pm 0.20$ & - & $24.75 \pm 0.08$ & 24.91 \\
\hline
\end{tabular}

a) not entrained

S18

S45

S51

\section{b) entrained}

$\mathrm{S} 17$

S31

S26

S62

- , not measured.

1988) with reagents obtained from Stockgrand Ltd (University of Surrey, UK). The limit of sensitivity (LOS) for the assay was $0.5 \mathrm{ng} / \mathrm{ml}$. The interassay coefficients of variation $(\mathrm{CV})$ were $19.0 \%$ at $3.8 \mathrm{ng} / \mathrm{ml}(n=26), 15.4 \%$ at $20.1 \mathrm{ng} / \mathrm{ml}(n=26)$ and $13.3 \%$ at $41.7 \mathrm{ng} / \mathrm{ml}(n=25)$.

Urinary cortisol was extracted with dichloromethane and measured by RIA (Riad-Fahmy et al. 1979) using an antiserum raised in sheep (Scottish Antiserum Production Unit, UK) and an iodinated radiolabel (Amersham International, UK). The LOS for the assay was $5.5 \mathrm{nmol} / \mathrm{l}$. The interassay $\mathrm{CV}$ was $14.9 \%$ at $49 \mathrm{nmol} / \mathrm{l}(n=22), 11.2 \%$ at $185 \mathrm{nmol} / \mathrm{l}(n=20)$ and $15.3 \%$ at $861 \mathrm{nmol} / \mathrm{l}(n=20)$.

\section{Results}

The mid-point of the crossing times correlated well with the acrophase times for both the aMT6s $(r=0.93)$ and cortisol $(r=0.94)$ rhythms. Taus derived from midpoint crossing times and the acrophase times also correlated well ( $r=0.96$ and 0.94 for aMT6s and cortisol, respectively). However for clarity, only taus derived from analyses of the acrophase times are quoted.

\section{Repeatability of tau}

The aMT6s (Lockley et al. 1997) and cortisol (Skene et al. 1999) rhythms of 5 subjects were assessed in a previous study using identical methodology. There was a good correlation between the taus measured in the two separate studies for both aMT6s $(r=0.96, n=5)$ and cortisol $(r=0.73, n=5)$.

\section{aMT6s and cortisol output}

The subjects' endogenous aMT6s output (mean \pm S.D.) ranged from $9.1 \pm 6.8$ to $22.0 \pm 4.3 \mu \mathrm{g} / 24 \mathrm{~h}$ and was within the range for sighted subjects (mean \pm 2 S.D. $=9.5 \pm 16.6 \mu \mathrm{g} / 24 \mathrm{~h}, n=517$; English \& Arendt, unpublished results). It increased on average 169-fold (range 67 to 256-fold) during treatment which was again similar to comparable sighted subjects (mean increase of 198-fold, range 92 to 392-fold; Middleton et al. 1997). These data confirmed the subjects' compliance with the treatment order. During melatonin treatment, urinary aMT6s levels never fell to within the endogenous aMT6s range. There was no significant effect of melatonin treatment on the subjects' endogenous mean $24 \mathrm{~h}$ cortisol output. There were no significant differences in the subjects' aMT6s and cortisol $24 \mathrm{~h}$ output between the previous studies (Lockley et al. 1997, Skene et al. 1999) and the current one ( $n=5, P>0.05)$.

\section{Effects of melatonin on the circadian system}

Table 2 shows the aMT6s taus before and/or after the melatonin treatment and the cortisol taus throughout the study for all subjects. Overall, the circadian system, as assessed from the cortisol rhythms, did not appear to change during the melatonin treatment in three subjects (S18, S45, S51) compared with before and/or after the treatment (Table 2a). Thus these subjects did not appear to be entrained by the melatonin treatment (Fig. 1). Although they were clearly freerunning, two subjects (S45 and S51) had large 95\% confidence limits associated with the cortisol taus during their melatonin treatment (Table 2a) due to the long study duration and low frequency of sampling.

Similarly during melatonin treatment, CBT data for S45 showed a free-running tau with large $95 \%$ confidence limits $(24.86 \pm 2.32 \mathrm{~h})$. However, the tau was very similar to the preand post-melatonin treatment measurements $(24.90 \pm 0.10 \mathrm{~h}$ and $24.88 \pm 0.20 \mathrm{~h}$, respectively). 


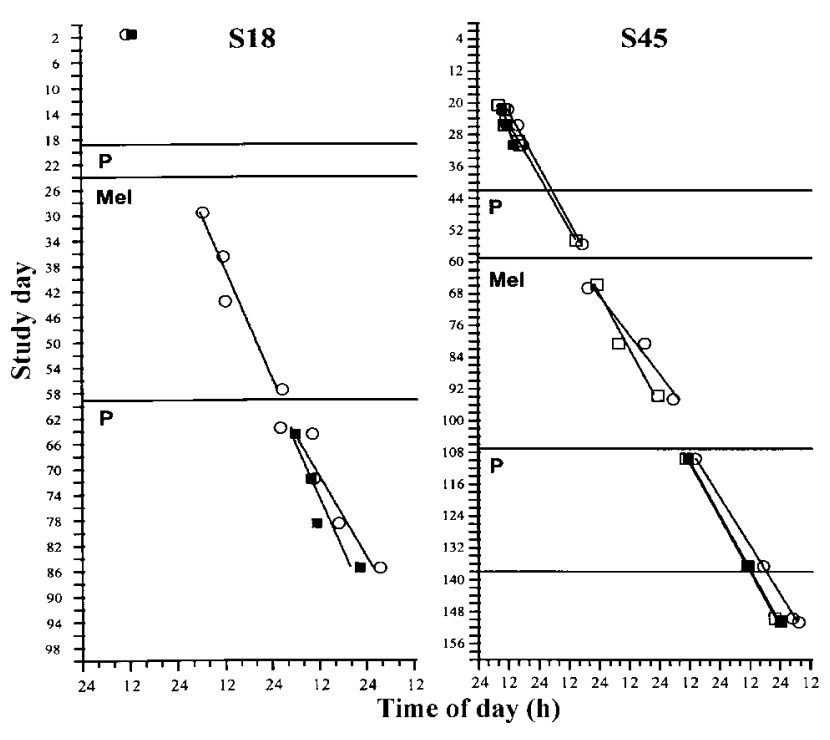

Figure 1 Acrophase times of the circadian phase markers for two subjects who did not entrain during melatonin treatment (S18, S45). Study days are shown on the vertical axis and the time of day is shown as a continuous sequence along the horizontal axis. Best-fit regression lines during each treatment are plotted for the aMT6s (ש), cortisol (O) and CBT ( $\square$ ) data. The calculated taus are shown in Table 2. Duration of the placebo $(\mathrm{P})$ and melatonin (Mel) is shown by the horizontal lines.

Three (S17, S31, S26) of the remaining four subjects exhibited a shortened cortisol tau which was statistically indistinguishable from entrainment (Table $2 b$ and Fig. 2). For these subjects, the mean ( \pm S.D.) cortisol acrophase times during melatonin treatment were $9.9 \pm 0.7 \mathrm{~h}(\mathrm{~S} 17), 12.9 \pm 1.2 \mathrm{~h}(\mathrm{~S} 31)$ and $11.5 \pm 1.5 \mathrm{~h}(\mathrm{~S} 26)$ which were within the range for normally entrained blind subjects (mean \pm 2 S.D. $=9.9 \pm 3.6 \mathrm{~h}, n=20$; Skene et al. 1999). In the remaining subject (S62), a tau could not be calculated during the melatonin treatment due to insufficient data points. The measured cortisol acrophase time $(12.4 \mathrm{~h})$ was consistent with a shortening of tau as the acrophase time, assuming a free-running cortisol rhythm, should have been 23.6 h. However, the lack of data points precludes a definitive assessment of melatonin's effects in this subject.

The circadian time (CT) at which melatonin administration commenced was calculated by extrapolation from the aMT6s tau (where melatonin onset time $=$ CT14 and aMT6s acrophase time $=$ CT21). The three subjects who showed entrainment began their melatonin treatment at CT11 (S17), CT12 (S26) and CT16 (S31). S62, with the advanced acrophase time, began melatonin treatment at CT11. The three subjects who did not entrain commenced their treatment at CT3 (S51), CT5 (S45) and CT19 (S18).

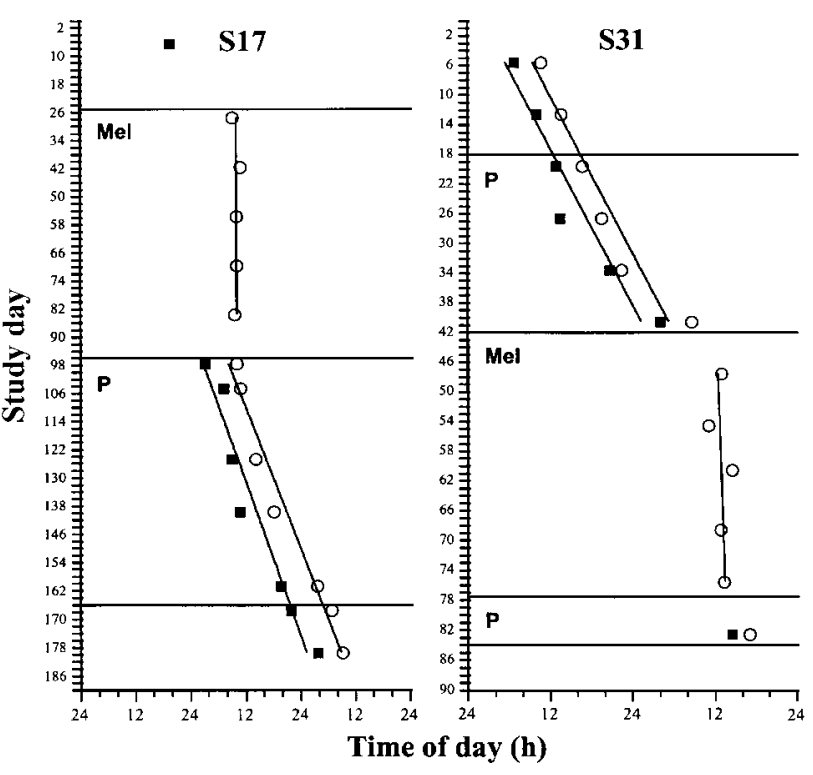

Figure 2 Acrophase times of the circadian phase markers for two subjects who entrained during melatonin treatment ( $\mathrm{S} 17$, S31). Other details as in Fig. 1.

\section{Discussion}

This study demonstrates for the first time that daily administration of $5 \mathrm{mg}$ melatonin can entrain the free-running circadian system of some blind subjects. The good degree of repeatability of the tau measurement between this and our previous studies allows any change in tau during the melatonin treatment to be considered significant.

Entrainment by melatonin has not been previously demonstrated in blind subjects. In the study by Sack et al. (1991), four out of five blind subjects showed phase advances in their melatonin $(n=4)$ and cortisol rhythm $(n=3)$ following melatonin treatment but entrainment was not observed. The reason for this is not clear but may have been due to the short duration of treatment (21 days). The other extensive study of melatonin's effect on phase in a blind man (Folkard et al. 1990) did not demonstrate any change in the CBT or cortisol rhythms during melatonin treatment.

The most recently published PRCs (Lewy \& Sack 1997, Middleton et al. 1997, Lewy et al. 1998) show that melatonin administration between approximately CT6 and CT18 (equivalent to $1300-0100 \mathrm{~h}$ clock time in a normally entrained subject) induces a phase advance and that between approximately CT18 and CT6 phase delays are predicted. Thus, in the present study, all the subjects who were entrained by melatonin began their treatment in an advance portion of the PRC whereas the three individuals who were not entrained, began melatonin treatment in the delay portion of the PRC.

These findings suggest that entrainment may be dependent on the phase at which melatonin treatment commences. The 
results are certainly intriguing and are consistent with studies of free-running sighted subjects in that entrainment only occurred when melatonin was started during an advance portion of the PRC and not when started during the delay phase (Middleton et al. 1997). To test this, further studies are underway to administer melatonin to the same subjects as reported here at the opposite circadian phase.

However, there may be some problems with this hypothesis. For example, why did the subjects in the present study not entrain once they reached the appropriate advance phase, as would be predicted from the PRC and animal experiments (Redman et al. 1983)? A similar lack of entrainment was observed in two sighted subjects by Middleton and colleagues (1997).

Another explanation for the subjects' lack of entrainment in our study may be that exogenous melatonin is affecting melatonin receptor sensitivity (Stetson et al. 1986, Gauer et al. 1993). In addition, beginning melatonin treatment in the delay part of the PRC may somehow compromise the ability of melatonin to entrain the circadian system by, for example, altering the PRC such that phase advances are no longer possible.

In the present study, it may be that the duration of melatonin treatment in the non-entrained subjects was not long enough to ensure a continued period of melatonin exposure within the advance portion of the PRC. It is also possible that some individuals are simply insensitive to melatonin (Zhdanova et al. 1995). There may be large individual differences in PRCs and some individuals may be insensitive to the constantly high circulating levels of melatonin during treatment.

From the one entrained subject (S17) who received melatonin before placebo, there appeared to be no 'after-effect' of melatonin on circadian periodicity. S17 free-ran after stopping melatonin treatment with a tau (aMT6s, 24.27士0.08 h) (Fig. 2) comparable to that measured in a previous study (24.34 \pm 0.10 h; Lockley et al. 1997).

Apart from the circadian phase of treatment onset, no other measured parameter was consistently different between subjects who did and those who did not entrain with melatonin. There were no significant differences between the age of the subjects, the duration of treatment, the time of year of treatment, their endogenous $24 \mathrm{~h}$ aMT6s production, their aMT6s concentrations following melatonin treatment or their taus.

An alternative hypothesis to explain these results would be that all of the subjects had shortened taus during melatonin treatment. It could be argued that the acrophases at the end of the melatonin treatment for S18 (day 57), S45 (day 94) and S51 (day 165, not shown) were advanced by $24 \mathrm{~h}$ rather than plotted free-running as shown. This alternative hypothesis requires that the melatonin treatment induced net phase advances in all three subjects which were greater than their endogenous delays thus making their rhythms go 'backwards'. This is unlikely given the sinusoidal shape of the PRC as, at the point where the induced advance equals the endogenous delay, the rhythms would be expected to stabilise. More frequent phase assessment in future studies will prevent this ambiguity.

In summary, the present study shows the first demonstration of entrainment of free-running blind subjects by melatonin treatment assessed using reliable physiological markers of the circadian system.

\section{Acknowledgements}

This work was supported by The Wellcome Trust (Grant 048197/Z/96/Z) and Stockgrand Ltd, University of Surrey, Guildford, UK.

\section{References}

Aldhous M \& Arendt J 1988 Radioimmunoassay for 6-sulphatoxymelatonin in urine using an iodinated tracer. Annals of Clinical Biochemistry 25 298-303.

Aldhous ME \& Arendt J 1991 Assessment of melatonin rhythms and the sleep-wake cycle in blind subjects. Advances in Pineal Research 5 307-309.

Arendt J, Aldhous M \& Wright J 1988 Synchronisation of a disturbed sleep-wake cycle in a blind man by melatonin treatment. Lancet $\mathbf{i}$ $772-773$.

Arendt J, Bojkowski C, Folkard S, Franey C, Marks V, Minors D, Waterhouse J, Wever RA, Wildgruber C \& Wright J $1985 a$ Some effects of melatonin and the control of its secretion in humans. In Photoperiodism, melatonin and the pineal (Ciba Foundation Symposium 117), pp 266-283. Eds DC Evered \& S Clark. London: Pitman.

Arendt J, Bojkowski C, Franey C, Wright J \& Marks V $1985 b$ Immunoassay of 6-hydroxymelatonin sulfate in human plasma and urine: abolition of the urinary 24-h rhythm with atenolol. Journal of Clinical Endocrinology and Metabolism 60 1166-1173.

Arendt J, Skene DJ, Middleton B, Lockley SW \& Deacon S 1997 Efficacy of melatonin treatment in jet lag, shift work, and blindness. Journal of Biological Rhythms 12 604-617.

Borbély AA 1982 A two process model of sleep regulation. Human Neurobiology 1 195-204.

Folkard S, Arendt J, Aldhous M \& Kennett H 1990 Melatonin stabilises sleep onset time in a blind man without entrainment of cortisol or temperature rhythms. Neuroscience Letters 113193 198.

Gauer F, Masson-Pevet M, Skene DJ, Vivien-Roels B \& Pevet P 1993 Daily rhythms of melatonin binding sites in the rat pars tuberalis and suprachiasmatic nuclei; evidence for a regulation of melatonin receptors by melatonin itself. Neuroendocrinology 57 120-126.

Hashimoto S, Nakamura K, Honma S \& Honma KI 1998 Free-running of plasma melatonin rhythm prior to full manifestation of a non-24 h sleep-wake syndrome. Psychiatry and Clinical Neurosciences 52 264-265.

Hayakawa T, Kamei Y, Urata J, Shibui K, Ozaki S, Uchiyama M \& Okawa M 1998 Trials of bright light and melatonin administration in a patient with non-24 h sleep-wake syndrome. Psychiatry and Clinical Neurosciences 52 261-262.

Kato M, Kajimura N, Sekimoto M, Watanabe \& Takahashi K 1998 Melatonin treatment for rhythm disorder. Psychiatry and Clinical Neurosciences 52 262-263. 
Lewy AJ, Ahmed S, Jackson JML \& Sack RL 1992 Melatonin shifts human circadian rhythms according to a phase-response curve. Chronobiology International 9 380-392.

Lewy AJ, Bauer VK, Ahmed S, Thomas KH, Cutler NL, Singer CM, Moffit MT \& Sack RL 1992 The human phase response curve (PRC) to melatonin is about $12 \mathrm{~h}$ out of phase with the PRC to light. Chronobiology International 15 71-83.

Lewy AJ \& Sack RL 1997 Exogenous melatonin's phase-shifting effects on the endogenous melatonin profile in sighted humans: A brief review and critique of the literature. Journal of Biological Rhythms 12 588-594.

Lockley SW, Skene DJ, Arendt J, Tabandeh H, Bird AC \& Defrance R 1997 Relationship between melatonin rhythms and visual loss in the blind. Journal of Clinical Endocrinology and Metabolism $\mathbf{8 2}$ 3763-3770.

Middleton B, Arendt J \& Stone BM 1997 Complex effects of melatonin on human circadian rhythms in constant dim light. Journal of Biological Rhythms 12 69-76.

Nelson W, Tong YL, Lee J-K \& Halberg F 1979 Methods for cosinorrhythmicity. Chronobiologica 6 305-323.

Okawa M, Uchiyama M, Ozaki S, Shibul K, Kamei Y, Hayakawa T \& Urata J 1998 Melatonin treatment for circadian rhythm sleep disorders. Psychiatry and Clinical Neurosciences 52 259-260.

Redman JR, Armstrong SM \& Ng KT 1983 Free-running activity rhythms in the rat: entrainment by melatonin. Science 219 10891091.

Riad-Fahmy D, Read GF, Gaskell SJ, Dyas J \& Hindawi R 1979 A simple direct radioimmunoassay for plasma cortisol, featuring a 125I-radioligand and a solid-phase separation technique. Clinical Chemistry 25 665-668.

Sack RL \& Lewy AJ 1997 Melatonin as a chronobiotic: treatment of circadian desynchrony in night workers and the blind. Journal of Biological Rhythms 12 595-603.

Sack RL, Lewy AJ, Blood ML, Keith LD \& Nakagawa H 1992 Circadian rhythm abnormalities in totally blind people: incidence and clinical significance. Journal of Clinical Endocrinology and Metabolism 75 127-134.
Sack RL, Lewy AJ, Blood ML, Stevenson J \& Keith LD 1991 Melatonin administration to blind people: phase advances and entrainment. Journal of Biological Rhythms 6 249-261.

Sack RL, Lewy AJ \& Hoban TM 1987 Free-running melatonin rhythms in blind people: Phase shifts with melatonin and triazolam administration. In Temporal Disorder in Human Oscillatory Systems, pp 219-224. Eds L Rensing, U Van der Heiden \& MC Mackey. Heidelberg: Springer-Verlag.

Sack RL, Stevenson J \& Lewy AJ 1990 Entrainment of a previously free-running blind human with melatonin administration. Sleep Research 19404.

Shanahan TL, Kronauer RE, Duffy JF, Williams GH \& Czeisler CA 1999 Melatonin rhythm observed throughout a three-cycle brightlight stimulus designed to reset the human circadian pacemaker. Journal of Biological Rhythms 14 237-253.

Skene DJ, Lockley SW, James K \& Arendt J 1999 Correlation between urinary cortisol and 6-sulphatoxymelatonin rhythms in field studies of blind subjects. Clinical Endocrinology 50 715-719.

Siebler M, Steinmetz H \& Freund H-J 1998 Therapeutic entrainment of circadian rhythm disorder by melatonin in a non-blind patient. Journal of Neurology 245 327-328.

Stetson MH, Sarafidis E \& Rollag MD 1986 Sensitivity of adult male Djungarian hamsters (Phodopus sungorus sungorus) to melatonin injections throughout the day: effects on the reproductive system and the pineal. Biological Reproduction 35 618-623.

Tzischinsky O, Pal I, Epstein R, Dagan Y \& Lavie P 1992 The importance of timing in melatonin administration in a blind man. Journal of Pineal Research 12 105-108.

Zaidan R, Geoffriau M, Brun J, Taillard J, Bureau C, Chazot G \& Claustrat B 1994 Melatonin is able to influence its secretion in humans: description of a phase-response curve. Neuroendocrinology 60 105-112.

Zhdanova IV, Wurtman RJ, Lynch HJ, Ives JR, Dollins AB, Morabito C, Matheson JK \& Schomer 1995 Sleep-inducing effects of low doses of melatonin ingested in the evening. Clinical Pharmacology and Therapeutics 57 552-558. 